\title{
Epidemiologic and Clinical Characteristics of COVID-19 Patients from a Quarantine Center in a Developing Community: A Retrospective Study
}

This article was published in the following Dove Press journal: International Journal of General Medicine

\author{
Wail N Khraise' \\ Tala W Khraise' \\ Bright Starling Emerald $\mathbb{D}^{2}$ \\ Mohammed Z Allouh (D) ${ }^{2,3}$ \\ 'Department of Anesthesia and \\ Recovery, Faculty of Medicine, Jordan \\ University of Science and Technology, \\ Irbid, Jordan; ${ }^{2}$ Department of Anatomy, \\ College of Medicine and Health Sciences, \\ United Arab Emirates University, Al Ain, \\ United Arab Emirates; ${ }^{3}$ Department of \\ Anatomy, Faculty of Medicine, Jordan \\ University of Science and Technology, \\ Irbid, Jordan
}

Purpose: Coronavirus disease 2019 (COVID-19) is an ongoing infectious disease caused by the severe acute respiratory syndrome coronavirus 2 (SARS-CoV-2) agent, and is generally known as novel coronavirus. The impact of the disease has had apparent effects on health, social, and economic levels. This study aimed to investigate the epidemiologic features, clinical presentations, laboratory examination, and outcomes of COVID-19 patients in Jordan.

Patients and Methods: This retrospective study included all COVID-19 patients admitted to the quarantine center at King Abdullah University Hospital in Jordan between March 16 and May 21, 2020. Patient information was abstracted from hospital electronic records and included patient demographics, symptoms, comorbidities, laboratory tests, infection period, and outcome. Results: Approximately $42 \%$ of the quarantined individuals were asymptomatic. The majority of symptomatic patients had mild to moderate symptoms of the disease. Only ten patients $(9.3 \%)$ had severe symptoms, with one of them progressed to a critical stage and died 15 days after admission. Erythrocyte sedimentation rate (ESR), lactate dehydrogenase (LDH), and D-dimer levels were significantly elevated in many patients. More than twothirds $(69.8 \%)$ of the symptomatic patients were females. Elevated ESR and LDH levels, along with longer periods of infection, were significantly associated with symptomatic patients.

Conclusion: A large proportion of Jordanian patients were asymptomatic carriers of the virus. This finding emphasizes the importance of a continuous surveillance process to discover these contagious carriers to control the disease spread. Additionally, we suggest that future analyses should investigate for sexual variation in the inflammatory response to this viral infection since most of our symptomatic patients were females. Finally, the role of ESR and LDH biomarkers should be investigated further for the possibility of earlier identification of patients with greater risk.

Keywords: coronavirus, COVID-19, infection, pandemic, respiratory, SARS

\section{Introduction}

Coronavirus disease 2019 (COVID-19) is an ongoing infectious disease caused by the severe acute respiratory syndrome coronavirus 2 (SARS-CoV-2) and is known generally as novel coronavirus. ${ }^{1}$ Since its detection in Wuhan, China, in December 2019, the pandemic has proliferated quickly worldwide, with unprecedented social, economic, and health effects. ${ }^{2}$ More importantly, many medical experts warn that the virus is unlikely to disappear, and the possibility of other waves of outbreak, especially during the winter, is very high. ${ }^{3}$
Correspondence: Mohammed Z Allouh
Department of Anatomy, College of Medicine and Health Sciences, United Arab Emirates University, Al Ain, United Arab Emirates

Tel +97 I37 I37 55I

Email m_allouh@uaeu.ac.ae

Wail N Khraise

Department of Anesthesia and Recovery, Faculty of Medicine, Jordan University of Science and Technology, Irbid, Jordan

Tel +962 79905 I295

Email wnkhraise@just.edu.jo

International Journal of General Medicine 2020:13 937-944 
The mortality rate of COVID-19 is estimated to be $4.1 \%,{ }^{4}$ which is much lower than the rate for other respiratory infections caused by similar viral strains. For example, the SARS mortality rate is $9.6 \%,{ }^{5}$ while that for Middle East Respiratory Syndrome (MERS) is $34.4 \%{ }^{6}$ However, the number of infected cases of COVID-19 is significantly higher than that of either SARS or MERS. While the number of SARS and MERS cases did not exceed a few thousands, the number of COVID-19 cases is about 35 million to date and still increasing. ${ }^{4}$ This is due to a longer incubation period, higher survival rate, and greater spread capability of the novel coronavirus. ${ }^{7}$ Additionally, the situation in countries where politics does not help impose proper quarantine measures has played a significant role in spreading the infection. For example, the number of infected cases in the United States and Brazil has reached about 7 and 5 million to date, respectively ( $\sim 34 \%$ of worldwide cases). ${ }^{4}$

The clinical course of the disease can be categorized into several stages based on disease severity. Several studies from different populations reported individuals who tested positive for SARS-CoV-2 and remained asymptomatic during their infection period. ${ }^{8-10}$ However, the asymptomatic persons are still considered contagious. ${ }^{10}$ The proportion of these asymptomatic cases has not yet been thoroughly evaluated. ${ }^{11}$ Furthermore, the World Health Organization has classified the symptomatic stages of COVID-19 disease into four main stages concerning the symptom severity (mild, moderate, severe, and critical). ${ }^{12}$ Most people with COVID-19 have only mild (40\%) or moderate $(40 \%)$ disease with symptoms, including cough, headache, fatigue, nasal congestion, and/or fever. ${ }^{12}$ Approximately $15 \%$ have severe disease with persistent fever and dyspnea that require oxygen support. The remaining 5\% have a critical disease with complications, such as respiratory failure, acute respiratory distress syndrome, sepsis, septic shock, thromboembolism, and/or multiorgan failure, including acute kidney injury and cardiac injury. ${ }^{12}$

To our knowledge, no comprehensive descriptive study exists to date on COVID-19 patients in Jordan. This study aimed to investigate the epidemiologic features, clinical presentations, laboratory examination, and outcomes of COVID-19 patients who were identified and quarantined in Northern Jordan during this pandemic period. The study provided a better understanding of the disease pathogenesis in our region. It helped to establish safer preventive measures, with more appropriate quarantine and treatment planning in case of a second wave strike of the virus.

\section{Patients and Methods Study Protocol}

In March 2020, the Ministry of Health in Jordan launched a general surveillance process to identify and quarantine individuals who were tested positive to the SARS-CoV-2 virus to control the spread of the infection. A special quarantine center and testing laboratory were established at King Abdullah University Hospital (KAUH) in Northern Jordan. Surveillance teams of health workers were tasked by the ministry to obtain nasopharyngeal swab samples from the general population in different towns and villages in Northern Jordan. Nasopharyngeal swabs were collected along with contact information and submitted to the testing laboratory at KAUH on a daily basis. Reverse transcriptase-polymerase chain reaction assays were performed on the swab specimens. The specimens were tested with the assay developed by the Centers for Disease Control, targeting the N1 and N2 genes. ${ }^{13}$ Individuals who were tested positive to the virus were immediately contacted and admitted by the health authority personnel to the quarantine center at KAUH.

This retrospective study included all individuals admitted to the quarantine center at KAUH from March 16, 2020, to May 21, 2020, after being tested positive for the virus as described earlier. The KAUH is a tertiary referral center in Northern Jordan, with 683 beds. This teaching hospital is affiliated with Jordan University of Science and Technology (JUST). It contains the only quarantine center for COVID-19 patients in Northern Jordan with a capacity of 200 beds. The hospital is the only referral center in Northern Jordan, serving approximately 2 million people. ${ }^{14}$ All COVID-19 patients admitted to the quarantine center at the hospital were detected during the aforementioned surveillance process performed by the public health authority.

The study was conducted with the approval of the institutional review board committee at KAUH and the deanship of research at JUST (\#408-2020), and in accordance with the Declaration of Helsinki and its later amendments for ethical research performance. Patient consents were not required because the data were used in aggregate with no personal identifiers. Data were abstracted from hospital electronic records and included patient demographics (ie, sex and age), comorbidities, laboratory tests, 
symptoms, infection period, and outcome. All laboratory test results were obtained within 24 hours of hospital admission. Laboratory tests included blood serum levels of hemoglobin $(\mathrm{Hb})$, creatinine, alanine transaminase (ALT), aspartate transaminase (AST), albumin, c-reactive protein, erythrocyte sedimentation rate (ESR), lactate dehydrogenase (LDH), and D-dimer molecule.

\section{Statistical Analyses}

Continuous numeric variables were expressed as the mean \pm standard deviation and median values. Categorical variables were described using the frequency numbers and percentages. Continuous variables were examined using the independent samples $t$-tests, and $P<0.05$ indicated a statistically significant difference. Categorical variables were examined using the one-sample and Pearson's $\chi^{2}$ tests of association. If a substantial $(P<0.05)$ association was found between categorical variables, then a post hoc residual analysis was conducted to determine the exact significance in the contingency table. Additionally, logistic regression analysis was used to determine the main predictors of symptomatic patients, and $P<0.05$ was considered statistically significant.

\section{Results}

\section{Patient Characteristics}

The study included 108 confirmed COVID-19 patients. Patient characteristics and clinical presentations are provided in Table 1. Female patients contributed to $58.3 \%$ of the study cohort. The patients' mean age was $36.4 \pm 17.1$ years, with the highest ratio (45.4\%) being young adults 20 to 40 years old. The most common comorbidities experienced were hypertension (17.6\%) and type II diabetes mellitus $(10.2 \%)$, as these are the two most common comorbidities among the Jordanian population.

Interestingly, a greater $(P<0.01)$ proportion of patients were asymptomatic (41.7\%) during their infection period. The most common presenting symptoms in symptomatic patients were dry cough and upper respiratory tract symptoms, including nasal congestion, runny nose, pain or pressure behind the face, and sore throat. The mean length of the infection period in our patient cohort was $13.5 \pm 7.6$ days.

The majority of our patients were asymptomatic or had mild to moderate symptoms. Only ten patients had severe symptoms with persistent fever, chills, cough, and difficulty breathing. Unfortunately, one patient (an 80-year-old female with breast cancer) with severe symptoms had progressed to a critical stage and died 15 days after admission.

\section{Laboratory Findings}

Most laboratory values were within normal ranges, except for ESR, LDH, and D-dimer, which had mean values higher than their normal ranges (Table 2). The distribution analysis revealed a higher $(P<0.01)$ number of patients with elevated ESR and LDH levels at admission. Notably, approximately three-quarters of the patients $(P<0.001$, 74.1\%) had elevated D-dimer blood levels (Table 3).

\section{Comparison Between Mild and Severely Symptomatic Patients}

A summary of the comparison between mild and severely symptomatic COVID-19 patients is provided in Table 4. Patients with severe symptoms were older $(\mathrm{P}<0.01)$ than patients with mild symptoms. Most of the patients with mild symptoms were young adults, whereas $80 \%$ of patients with severe symptoms were over $40 \mathrm{y}$ of age (old adults and seniors). Additionally, more comorbidities were associated with severely-symptomatic patients, and a higher $(P<0.05)$ frequency of malignancy was detected in patients with severe COVID-19 symptoms compared to ones with mild symptoms.

Regarding the laboratory findings, more severelysymptomatic patients $(P<0.05)$ had elevated C-reactive protein levels compared to patients with mild symptoms. The increases in the percentages of ESR, LDH, and D-dimer elevated values in patients with severe symptoms did not reach significant levels. There was no significant difference in the infection period. However, the mortality rate was higher $(P<0.05)$ in patients with severe symptoms compared to patients with mild ones (Table 4).

\section{Comparison Between Symptomatic and Asymptomatic Patients}

A summary of the comparison between symptomatic and asymptomatic COVID-19 patients is provided in Table 5 . There was a significant association between the patient sex and the appearance of symptoms since more than twothirds of the symptomatic patients were females (69.8\%). However, there was no significant association between the patient's age and the symptomatic condition. Smoking also was not significantly associated with patient symptoms.

Regarding the laboratory tests, ESR was the only test to be significantly associated with the patients' symptomatic 
Table I Characteristics of Patients with Novel Coronavirus Infection in Northern Jordan

\begin{tabular}{|c|c|c|}
\hline \multirow[t]{2}{*}{ Associated Variables } & Number & Percent (\%) \\
\hline & \multicolumn{2}{|c|}{ Mean \pm SD } \\
\hline \multicolumn{3}{|l|}{ Sex } \\
\hline Male & 45 & 41.7 \\
\hline Female & 63 & 58.3 \\
\hline Age (y) & \multicolumn{2}{|c|}{$36.4 \pm 17.1$} \\
\hline \multicolumn{3}{|l|}{ Age category } \\
\hline Children and adolescents ( $1-19$ y) & 16 & 14.8 \\
\hline Young adults $(20-40 y)$ & 49 & $45.4^{* *}$ \\
\hline Old adults $(4 \mid-60 y)$ & 34 & 31.5 \\
\hline Seniors $(6 \mathrm{I}-80 \mathrm{y})$ & 9 & 8.3 \\
\hline Smoking & 26 & 24.1 \\
\hline \multicolumn{3}{|l|}{ Comorbidities } \\
\hline Ischemic heart disease & 5 & 4.6 \\
\hline Hypertension & 19 & 17.6 \\
\hline Diabetes mellitus & 11 & 10.2 \\
\hline Dyslipidemia & 7 & 6.5 \\
\hline Malignancy & 2 & 1.9 \\
\hline \multicolumn{3}{|l|}{ Symptoms } \\
\hline Asymptomatic & 45 & $41.7 * *$ \\
\hline Cough & 43 & $39.8 * *$ \\
\hline Headache & 14 & 13.0 \\
\hline Fever & 16 & 14.8 \\
\hline Chills & 10 & 9.3 \\
\hline URTS & 47 & $43.5^{* *}$ \\
\hline Dyspnea & 14 & 13.0 \\
\hline Infection period (days) & \multicolumn{2}{|l|}{$13.5 \pm 7.6$} \\
\hline \multicolumn{3}{|l|}{ Outcome } \\
\hline Cured & 107 & $99.1 * *$ \\
\hline Dead & I & 0.9 \\
\hline
\end{tabular}

Notes: ${ }^{* * P}<0.01$, chi-square test.

Abbreviations: ALT, alanine transaminase; AST, aspartate transaminase; ESR, erythrocyte sedimentation rate; URTS; upper respiratory tract symptoms; SD, standard deviation; $y$, years.

state. A higher $(P<0.001)$ mean value of ESR was found in the symptomatic patients. Compared to asymptomatic patients, significantly more symptomatic patients $(60 \% ; P<$ 0.01) had elevated ESR levels (Table 5). Finally, a significant association was detected between the infection period and symptomatic condition of the patients. A longer $(P<0.001)$ period of infection was observed in symptomatic compared to asymptomatic patients (Table 5).

Furthermore, a binary logistic regression model that included all variables in Table 5 was applied. The two main predictors for symptomatic patients were the infection period and LDH test results. Regression analysis revealed that patients with high LDH values had a higher risk (4.1 times) of being symptomatic than those with normal LDH values $(P<0.05)$. Additionally, symptomatic patients showed a longer (1.1 times) infection period than asymptomatic patients $(P<0.05)$.

\section{Discussion}

To our knowledge, this is the largest and most comprehensive evaluation of COVID-19 patients from Jordan. Previous studies included fewer samples, were limited to epidemiologic findings only, and were focused on one field of medical specialty. ${ }^{15,16}$ Our study uniquely revealed the characteristic differences between symptomatic and asymptomatic patients with novel coronavirus infection. Additionally, it provided important information about the role of the inflammatory and coagulopathy biomarkers during the disease course.

Approximately half of the infected people were young adults between 20 and 40 years old, presumably, because these are the most socioeconomically active individuals. Additionally, the study revealed that many infected individuals were asymptomatic during their entire infection period. These individuals were actually silent carriers of the virus rather than genuine patients. A large proportion of asymptomatic individuals were males. On the other hand, the majority of symptomatic patients $(\sim 70 \%)$ were females. This finding probably could be attributed to the more intense immune response in females, which leads to more symptomatic complications than those in males. ${ }^{17,18}$ This sex-based disparity in immune response may be attributed to differences in sex hormones. Generally, testosterone has an immunosuppressive effect, while estrogen has an immunoenhancing effect. ${ }^{19-21}$ For example, estrogen has been linked with enhanced capability of producing antibodies. ${ }^{18}$

Mean values of the inflammatory and disease biomarkers ESR and LDH were above the normal range in our COVID-19 patients since a large number of patients had elevated blood levels for these two biomarkers. This finding is comparable to several previous studies from different populations. ${ }^{22-24}$ Additionally, ESR was significantly higher in the symptomatic compared to asymptomatic patients. The ESR level reportedly is positively associated with the severity of COVID-19 infection. ${ }^{25}$ Previous studies reported significantly higher ESR values in severe and critically ill patients than those in mild and moderately ill patients. $^{26,27}$ The higher ESR values in our symptomatic patients suggested that the inflammatory response was more prominent in them than in the asymptomatic patients. 
Table 2 Mean Laboratory Test Values for COVID-19 Patients in Northern Jordan

\begin{tabular}{|l|l|l|l|}
\hline Test & Normal Range & Mean \pm SD & Median \\
\hline Leukocytes $\left(\times 10^{3} / \mathrm{mm}^{3}\right)$ & $4-1 \mathrm{I}$ & $7.0 \pm 2.6$ & 6.8 \\
Neutrophils $\left(\times 10^{3} / \mathrm{mm}^{3}\right)$ & $1.5-7$ & $4.4 \pm 2.5$ & 4.0 \\
Lymphocytes $\left(\times 10^{3} / \mathrm{mm}^{3}\right)$ & $1-3$ & $2.0 \pm 0.9$ & 1.9 \\
Platelets $\left(\times 10^{3} / \mathrm{mm}^{3}\right)$ & $150-400$ & $231.0 \pm 64.7$ & 220 \\
Hemoglobin $(\mathrm{g} / \mathrm{dL})$ & $11.5-15.5$ & $13.8 \pm 1.9$ & 13.9 \\
Creatinine $(\mu \mathrm{mol} / \mathrm{L})$ & $50-107$ & $67.6 \pm 19.5$ & 64.5 \\
ALT $(\mathrm{U} / \mathrm{L})$ & $<33$ & $21.2 \pm 14.9$ & 16 \\
AST $(\mathrm{U} / \mathrm{L})$ & $<32$ & $21.8 \pm 7.4$ & 20 \\
Albumin $(\mathrm{g} / \mathrm{L})$ & $35-55$ & $45.5 \pm 3.9$ & 45.8 \\
C-reactive protein $(\mathrm{mg} / \mathrm{L})$ & $<10$ & $6.7 \pm 8.4$ & 2.9 \\
ESR $(\mathrm{mm} / \mathrm{h})$ & $<20$ & $21.9 \pm 17.9 \uparrow$ & 20 \\
LDH $(\mathrm{U} / \mathrm{L})$ & $260-420$ & $447.8 \pm 145.9 \uparrow$ & \\
D-dimer $(\mu \mathrm{gg} / \mathrm{mL})$ & $<0.5$ & $1.0 \pm 0.4 \uparrow$ & \\
\hline
\end{tabular}

Note: ${ }^{\uparrow}$ indicates elevated mean value.

Abbreviations: ALT, alanine transaminase; AST, Aspartate transaminase; ESR, erythrocyte sedimentation rate; LDH, lactate dehydrogenase; SD, standard deviation; $y$, years.

Table 3 Distribution of Laboratory Test Results for Patients with Novel Coronavirus Infection in Northern Jordan

\begin{tabular}{|c|c|c|c|}
\hline Test & Low $\mathbf{N}(\%)$ & Normal N (\%) & High N (\%) \\
\hline Leukocytes (108/108) & $15(13.9)$ & $85(78.7)$ & $8(7.4)$ \\
\hline Neutrophils (108//08) & $9(8.3)$ & $88(81.5)$ & II (I0.2) \\
\hline Lymphocytes (108/I08) & $8(7.4)$ & $82(75.9)$ & $18(16.7)$ \\
\hline Platelets (108//08) & $16(14.8)$ & $91(84.3)$ & $\mathrm{I}(0.9)$ \\
\hline Hemoglobin $(108 / 108)$ & $13(12.0)$ & $70(64.8)$ & $25(23.1)$ \\
\hline Creatinine $(106 / 108)$ & II (I0.4) & $89(84.0)$ & $6(5.6)$ \\
\hline ALT (I08//08) & - & $93(86.1)$ & $15(13.9)$ \\
\hline AST $(108 / 108)$ & - & $98(90.7)$ & $10(9.3)$ \\
\hline Albumin $(108 / 108)$ & $2(1.9)$ & $98(90.7)$ & $8(7.4)$ \\
\hline C-reactive protein $(102 / 108)$ & - & $74(72.5)$ & $28(27.5)$ \\
\hline ESR $(98 / 108)$ & - & $51(52.0)$ & $47(48.0)^{* *}$ \\
\hline LDH (94/108) & $0(0.0)$ & $48(51.1)$ & $46(48.9)^{* *}$ \\
\hline D-dimer $(103 / 108)$ & - & $23(22.3)$ & $80(77.7)^{* * *}$ \\
\hline
\end{tabular}

Notes: $* * \mathrm{P}<0.01$, ***P $<0.001$, chi-square test.

Abbreviations: ALT, alanine transaminase; AST, Aspartate transaminase; ESR, erythrocyte sedimentation rate; LDH, lactate dehydrogenase; N, number..

This also was supported by the longer infection period found in symptomatic than in asymptomatic patients.

D-dimer is a degraded protein fragment found in the blood after the fibrinolysis of a blood clot. ${ }^{28} \mathrm{D}$-dimer is normally undetectable in the blood unless there are formation and breakdown of blood clots. This allows a D-dimer serum test to be used to rule out thrombotic episodes and to help in early diagnosis of several thromboembolic conditions, including deep vein thrombosis, pulmonary embolism, and disseminated intravascular coagulation. ${ }^{29,30}$ Original studies suggested a hypercoagulable state in COVID-19 patients, as thromboembolism formation was reported by pathologic studies based on autopsies or biopsies for COVID-19 patients. ${ }^{31,32}$ Based on the previous fact, several researchers had linked the increase in D-dimer level with the hypercoagulable state in COVID19 patients. ${ }^{33,34}$ However, other researchers propose that elevated D-dimer levels could be associated with the inflammation process and not necessarily related to the thromboembolism condition in COVID-19 patients. $^{35}$ In fact, the exact reasons responsible for the elevated D-dimer levels in COVID-19 patients are only partially explained, and further investigations are warranted.

Comparing the findings of this study with other countries' findings revealed population-wise variations in sociodemographic characteristics and clinical presentation 
Table 4 Factors Associated with the Severity of Symptoms in COVID-19 Patients in Jordan

\begin{tabular}{|c|c|c|c|}
\hline Associated Variables & Mild Symptoms & Severe Symptoms & $P$-value \\
\hline Sex, n (\%) & & & 0.460 \\
\hline Male & $15(28.3)$ & $4(40.0)$ & \\
\hline Female & $38(71.7)$ & $6(60.0)$ & \\
\hline Mean age $(y)$, mean $\pm S D$ & $34.2 \pm 15.5$ & $50.3 \pm 18.4$ & 0.005 \\
\hline Age category, n (\%) & & & 0.046 \\
\hline Children and adolescents $(1-19 y)$ & $10(18.9)$ & I $(10.0)$ & \\
\hline Young adults $(20-40 \mathrm{y})$ & $23(43.4)^{\uparrow}$ & $\mathrm{I}(10.0)$ & \\
\hline Old adults $(4 \mid-60 y)$ & $16(30.2)$ & $6(60.0)$ & \\
\hline Seniors $(6 \mathrm{I}-80 \mathrm{y})$ & $4(7.5)$ & $2(20.0)$ & \\
\hline \multicolumn{4}{|l|}{ Comorbidities, n (\%) } \\
\hline Ischemic heart disease & $2(3.8)$ & $I(10.0)$ & 0.396 \\
\hline Hypertension & $7(13.2)$ & $2(20.0)$ & 0.573 \\
\hline Diabetes mellitus & $4(7.5)$ & $2(20.0)$ & 0.219 \\
\hline Dyslipidemia & $4(7.5)$ & $\mathrm{I}(10.0)$ & 0.792 \\
\hline Malignancy & $0(0.0)$ & $\mathrm{I}(10.0)^{\uparrow}$ & 0.020 \\
\hline \multicolumn{4}{|l|}{ Elevated Laboratory Values, n (\%) } \\
\hline C-reactive protein & $14(26.4)$ & $6(60.0)^{\uparrow}$ & 0.036 \\
\hline ESR & $29(54.7)$ & $8(80.0)$ & 0.136 \\
\hline LDH & $26(49.1)$ & $7(70.0)$ & 0.224 \\
\hline D-dimer & $39(73.6)$ & $9(90.0)$ & 0.264 \\
\hline Infection period (days), mean \pm SD & $16.2 \pm 7.7$ & $14.2 \pm 6.7$ & 0.572 \\
\hline Mortality, n (\%) & $0(0.0)$ & $\mathrm{I}(10.0)^{\uparrow}$ & 0.020 \\
\hline
\end{tabular}

Note: ${ }^{\uparrow}$ Higher than expected frequency.

Abbreviations: ESR, erythrocyte sedimentation rate; LDH, lactate dehydrogenase; NS, not significant; n, number; P, probability; SD, standard deviation; $y$, years.

of COVID-19 patients. For example, the mean age of the infected individuals in our study was 36.4 years, while the mean age from a study in Spain was 64.7 years. $^{36}$ Additionally, studies from Spain and China revealed that most of the patients were symptomatic. ${ }^{38,39}$ However, the majority of the patients in our study were asymptomatic or with mild symptoms. This is probably due to the younger age of our patients. Nevertheless, these comparison findings could justify the surveillance and quarantine approach adopted by the Jordanian authorities for the early identification of asymptomatic younger patients to control the spread of the disease.

Several limitations, which are mainly related to the methodology part of this research, should be considered. First, the study was conducted in a single quarantine center; therefore, the external validity of the study findings is limited to the assigned population of Northern Jordan. Second, the descriptive nature of the study prevents the acceptance of casual associations. Third, we were unable to determine the exact period of infection. Since patients were discovered during routine surveillance, it is possible that they acquired the infection several days before the detection test. Fourth, specific inflammatory test values such as the cluster of differentiation 4 and interferongamma were not reported for most patients, so they were excluded from the study. Finally, some laboratory test values were missing from the hospital record for few patients. However, we believe this issue does not affect the laboratory findings of the study.

\section{Conclusions}

In conclusion, this study revealed that a large proportion $(>40 \%)$ of COVID-19 patients in Jordan were indolent carriers of the virus. This emphasized the importance of a continuous surveillance process in order to control the spread of the disease. It also showed a sexual variation in the inflammatory response to the disease, as more than two-thirds of symptomatic patients were females. The symptomatic patients suffered a longer period of infection than asymptomatic carriers. Lastly, the main associated biomarkers with the disease course and severity were ESR and LDH inflammatory biomarkers. Further 
Table 5 Factors Associated with Symptomatic COVID-19 Patients in Northern Jordan

\begin{tabular}{|c|c|c|c|}
\hline Associated Variables & Symptomatic & Asymptomatic & $P$-value \\
\hline Sex, n (\%) & & & 0.004 \\
\hline Male & $19(30.2)$ & $26(57.8)$ & \\
\hline Female & $44(69.8)^{\uparrow}$ & $19(42.2)$ & \\
\hline Mean age $(y)$, mean $\pm S D$ & $36.8 \pm 16.9$ & $35.9 \pm 17.7$ & 0.797 \\
\hline Age category, n (\%) & & & 0.347 \\
\hline Children and adolescents (I-19y) & II (I7.5) & $5(11.1)$ & \\
\hline Young adults $(20-40 \mathrm{y})$ & $24(38.1)$ & $25(55.6)$ & \\
\hline Old adults $(4 \mid-60 y)$ & $22(34.9)$ & $12(26.7)$ & \\
\hline Seniors $(6 \mathrm{I}-80 \mathrm{y})$ & $6(9.5)$ & $3(6.7)$ & \\
\hline Smoking, n (\%) & & & 0.070 \\
\hline Yes & II (I7.5) & $15(33.3)$ & \\
\hline No & $52(82.5)$ & $30(66.7)$ & \\
\hline ESR value, mean $\pm S D$ & $26.3 \pm 18.7$ & $15.0 \pm 14.1$ & 0.001 \\
\hline ESR level, n (\%) & & & 0.003 \\
\hline Normal & $24(40.0)$ & $27(7 \mid . I)^{\uparrow}$ & \\
\hline High & $36(60.0)^{\uparrow}$ & II (28.9) & \\
\hline LDH value, mean \pm SD & $468.9 \pm|7| .2$ & $423.3 \pm 106.2$ & 0.138 \\
\hline LDH level, n (\%) & & & 0.059 \\
\hline Normal & $22(42.3)$ & $26(61.9)$ & \\
\hline High & $30(57.7)$ & $16(38.1)$ & \\
\hline D-dimer value, mean $\pm S D$ & $\mathrm{I} . \mathrm{I} \pm 0.5$ & $1.0 \pm 0.3$ & 0.370 \\
\hline D-dimer level, n (\%) & & & 0.773 \\
\hline Normal & $14(23.3)$ & $9(20.9)$ & \\
\hline High & $46(76.7)$ & $34(79.1)$ & \\
\hline Infection period (days), mean \pm SD & $16.0 \pm 7.6$ & $10.3 \pm 6.3$ & $<0.000$ \\
\hline
\end{tabular}

Note: ${ }^{\uparrow}$ Higher than expected frequency.

Abbreviations: ESR, erythrocyte sedimentation rate; LDH, lactate dehydrogenase; NS, not significant; n, number; P, probability; SD, standard deviation; $y$, years.

investigations are warranted to assess the role of these biomarkers in stratifying patients with greater risk of COVID-19.

\section{Disclosure}

The authors report no conflicts of interest in this work.

\section{References}

1. Ahn DG, Shin HJ, Kim MH, et al. Current status of epidemiology, diagnosis, therapeutics, and vaccines for novel coronavirus disease 2019 (COVID-19). J Microbiol Biotechnol. 2020;30:313-324. doi:10.4014/jmb.2003.03011

2. Bogoch II, Watts A, Thomas-Bachli A, Huber C, Kraemer MUG, Khan K. Pneumonia of unknown aetiology in Wuhan, China: potential for international spread via commercial air travel. $J$ Travel Med. 2020;27:taaa008.
3. Cyranoski D. We need to be alert': scientists fear second coronavirus wave as China's lockdowns ease. Nature. 2020. doi:10.1038/d41586020-00938-0

4. World Health Organization. Coronavirus disease (COVID-19) pandemic. Available from: https://www.who.int/emergencies/dis eases/novel-coronavirus-2019. Accessed October 4, 2020.

5. World Health Organization. Summary of probable SARS cases with onset of illness from November 12002 to July 31 2003. Available from: https:// www.who.int/csr/sars/country/table2004_04_21/en/. Accessed October 4, 2020.

6. World Health Organization. Middle East respiratory syndrome coronavirus (MERS-CoV) monthly summary, November 2019. Available from: https://www.who.int/emergencies/mers-cov/en/. Accessed October 4, 2020.

7. Jin Y, Yang H, Ji W, et al. Virology, epidemiology, pathogenesis, and control of COVID-19. Viruses. 2020;12:372.

8. Chen J, Qi T, Liu L, et al. Clinical progression of patients with COVID-19 in Shanghai, China. J Infect. 2020;80:e1-e6.

9. Choi MH, Ahn H, Ryu HS, et al. Clinical characteristics and disease progression in early-stage COVID-19 patients in South Korea. J Clin Med. 2020;9:1959. 
10. Rothe C, Schunk M, Sothmann P, et al. Transmission of 2019-nCoV infection from an asymptomatic contact in Germany. $N$ Engl J Med. 2020;382:970-971.

11. Nahshon C, Bitterman A, Haddad R, Hazzan D, Lavie O. Hazardous postoperative outcomes of unexpected COVID-19 infected patients: a call for global consideration of sampling all asymptomatic patients before surgical treatment. World J Surg. 2020;44(8):2477-2481.

12. World Health Organization. Clinical management of COVID-19. Interim guidance. (C) CC BY-NC-SA 3.0 IGO, 2020, p. 9. Available from: https://www.who.int/publications/i/item/clinical-managementof-covid-19.

13. Nalla AK, Casto AM, Huang MW, et al. Comparative performance of SARS-CoV-2 detection assays using seven different primer-probe sets and one assay kit. J Clin Microbiol. 2020;58:e0557-20.

14. Ministry of Planning and International Cooperation in Jordan. Estimated population of the kingdom by municipality and sex, at end-year 2018. Jordan; 2018. http://dosweb.dos.gov.jo/DataBank/ Population_Estimares/Municipalities.pdf.

15. Yusef D, Hayajneh W, Awad S, et al. Large outbreak of coronavirus disease among wedding attendees, Jordan. Emerg Infect Dis. 2020:26. Epub ahead of print. PMID: 32433907.

16. Alsharaydeh I, Rawashdeh H, Saadeh N, Obeidat B, Obeidat N. Challenges and solutions for maternity and gynecology services during the COVID-19 crisis in Jordan. Int $J$ Gynaecol Obstet. 2020;150:159-162.

17. Moulton VR. Sex hormones in acquired immunity and autoimmune disease. Front Immunol. 2018;9:2279.

18. Taneja V. Sex hormones determine immune response. Front Immunol. 2018;9:1931.

19. Markle JG, Fish EN. Sexx matters in immunity. Trends Immunol. 2014;35:97-104.

20. Klein SL. The effects of hormones on sex differences in infection: from genes to behavior. Neurosci Biobehav Rev. 2000;24:627-638.

21. Mo R, Chen J, Grolleau-Julius A, Murphy HS, Richardson BC, Yung RL. Estrogen regulates CCR gene expression and function in T lymphocytes. J Immunol. 2005;174:6023-6029.

22. de Sanctis V, Canatan D, Corrons JLV, et al. Preliminary Data on COVID-19 in patients with hemoglobinopathies: A multicentre ICET-A study. Mediterr J Hematol Infect Dis. 2020;12:e2020046.

23. Mardani R, Ahmadi Vasmehjani A, Zali F, et al. Laboratory parameters in detection of COVID-19 patients with positive RT-PCR; a diagnostic accuracy study. Arch Acad Emerg Med. 2020;8:e43.

24. Chen N, Zhou M, Dong X, et al. Epidemiological and clinical characteristics of 99 cases of 2019 novel coronavirus pneumonia in Wuhan, China: a descriptive study. Lancet. 2020;395:507-513.
25. Lapić I, Rogić D, Plebani M. Erythrocyte sedimentation rate is associated with severe coronavirus disease 2019 (COVID-19): a pooled analysis. Clin Chem Lab Med. 2020;58:1146-1148.

26. Wang D, Li R, Wang J, et al. Correlation analysis between disease severity and clinical and biochemical characteristics of 143 cases of COVID-19 in Wuhan, China: a descriptive study. BMC Infect Dis. 2020;20:519.

27. Yuan X, Huang W, Ye B, et al. Changes of hematological and immunological parameters in COVID-19 patients. Int $J$ Hematol. 2020;1-7.

28. Jacobs B, Obi A, Wakefield T. Diagnostic biomarkers in venous thromboembolic disease. J Vasc Surg Venous Lymphat Disord. 2016;4:508-517.

29. Schaefer JK, Jacobs B, Wakefield TW, Sood SL. New biomarkers and imaging approaches for the diagnosis of deep venous thrombosis. Curr Opin Hematol. 2017;24:274-281.

30. Le Gal G, Bounameaux H. D-dimer for the diagnosis of pulmonary embolism: a call for sticking to evidence. Intensive Care Med. 2005;31:1-2.

31. Xu Z, Shi L, Wang Y, et al. Pathological findings of COVID-19 associated with acute respiratory distress syndrome. Lancet Respir Med. 2020;8:420-422.

32. Lang Z, Zhang L, Zhang S, et al. Pathological study on severe acute respiratory syndrome. Chin Med J. 2003;116:976-980.

33. Zhou F, Yu T, Du R, et al. Clinical course and risk factors for mortality of adult inpatients with COVID-19 in Wuhan, China: a retrospective cohort study. Lancet. 2020;395:1054-1062.

34. Wright FL, Vogler TO, Moore EE, et al. Fibrinolysis shutdown correlation with thromboembolic events in severe COVID-19 infection. J Am Coll Surg. 2020;231:193-203.

35. Yu B, Li X, Chen J, et al. Evaluation of variation in D-dimer levels among COVID-19 and bacterial pneumonia: a retrospective analysis. J Thromb Thrombolysis. 2020:1-10. Epub ahead of print. PMID: 32524516

36. Rivera-Izquierdo M, Valero-Ubierna MDC, R-delAmo JL, et al. Sociodemographic, clinical and laboratory factors on admission associated with COVID-19 mortality in hospitalized patients: A retrospective observational study. PLoS One. 2020;15:e0235107.

37. Rivera-Izquierdo M, Valero-Ubierna MDC, Martínez-Diz S, et al. Clinical factors, preventive behaviours and temporal outcomes associated with COVID-19 infection in health professionals at a spanish hospital. Int J Environ Res Public Health. 2020;17:4305.

38. Guan WJ, Ni ZY, Hu Y, et al. Clinical characteristics of coronavirus disease 2019 in China. N Engl J Med. 2020;382:1708-1720.

39. Wang D, Hu B, Hu C, et al. Clinical characteristics of 138 hospitalized patients with 2019 novel coronavirus-infected pneumonia in Wuhan, China. JAMA. 2020;323:1061-1069.
International Journal of General Medicine

\section{Publish your work in this journal}

The International Journal of General Medicine is an international, peer-reviewed open-access journal that focuses on general and internal medicine, pathogenesis, epidemiology, diagnosis, monitoring and treatment protocols. The journal is characterized by the rapid reporting of reviews, original research and clinical studies across all disease areas. The manuscript management system is completely online and includes a very quick and fair peer-review system, which is all easy to use. Visit http://www.dovepress.com/ testimonials.php to read real quotes from published authors. 